\title{
Geometrical aspects of a generalized statistical mechanics
}

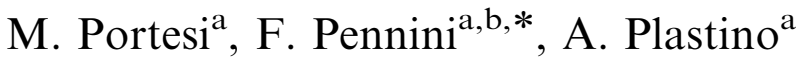 \\ ${ }^{a}$ Instituto de Física La Plata (IFLP, CONICET-UNLP) and Departamento de Fisica, Fac. de Cs. Exactas, \\ Universidad Nacional de La Plata, C.C. 67, 1900 La Plata, Argentina \\ ${ }^{\mathrm{b}}$ Departamento de Física, Universidad Católica del Norte, Casilla 1280, Antofagasta, Chile
}

Received 29 December 2005

Available online 14 June 2006

\begin{abstract}
We discuss here the use of generalized forms of entropy, taken as information measures, to characterize phase transitions and critical behavior in thermodynamic systems. Our study is based on geometric considerations pertaining to the space of parameters that describe statistical mechanics models. The thermodynamics of the system is the focus of attention in this geometric context.
\end{abstract}

(C) 2006 Elsevier B.V. All rights reserved.

Keywords: Information geometry; Nonextensive statistical mechanics; Generalized $q$-divergence

\section{Introduction}

Our aim is to add a note on current efforts to investigate the geometrical structure of the space of thermodynamic parameters for classical and quantum systems. This kind of studies has been developed by many authors (see, for instance, Refs. [1-9]), and has been applied to the ideal gas, the van der Waals gas, and magnetic systems that exhibit phase transitions. In such instances the Riemannian scalar curvature $R$ of the parameters' space plays such an important role in detecting critical behavior that it has been interpreted as a measure of the stability of the thermodynamic system (typically, for noninteracting models one obtains $R=0$, i.e., a flat geometry, while $R$ diverges at the critical point for interacting systems).

In Ref. [1] Weinhold considered the thermodynamic surface given by the fundamental relation $U=U\left(\left\{X_{i}\right\}\right)$ in the $(r+1)$-dimensional Gibbs space (with coordinates labelled by $U, X_{1}, \ldots, X_{r}$ ), and obtained the components of the metric tensor of that space as the second derivatives of the internal energy $U$ with respect to each pair of the $r$ extensive parameters $X_{i}$. Ruppeiner [2] focused attention on fluctuations of the thermodynamic magnitudes and obtained the metric tensor via second moments of the fluctuations. The two ensuing metrics have been proven by Mrugała to be equivalent [3]. Another statistical path for reaching a Riemannian metric [10-12] in the space of thermodynamic parameters is that originated in the works by Rao [13] and Amari (see, for instance, [14]) in the field of statistical mathematics, and also by Ingarden [4], Janyszek [5], and other authors in the field of thermodynamics and statistical mechanics. The concomitant

*Corresponding author. Departamento de Física, Universidad Católica del Norte, Casilla 1280, Antofagasta, Chile.

E-mail addresses: portesi@fisica.unlp.edu.ar (M. Portesi), fpennini@ucn.cl (F. Pennini), plastino@fisica.unlp.edu.ar (A. Plastino). 
information-theoretic approach is based on the concept of relative entropy and given in terms of the Boltzmann-Gibbs-Shannon entropy.

Also deserving mention are some recent interesting efforts in the field of information geometry, related to the generalized, nonextensive formulation of statistical mechanics [15,16]. Among these studies one finds the contributions by Amari et al. ([14] among others) in connection with the geometrical structure in the manifold of probability distributions, and the analysis by Abe [17] of the geometry of escort distributions, in connection with bit-variance and fluctuations of the crowding index of a multifractal. A new geometrical approach to thermo-statistical mechanics is introduced in Ref. [18], where the relevance of the approach within the contexts of nonextensive statistical thermodynamics is analyzed, showing that Riemannian geometry concepts yield a powerful tool. More recently, Naudts [19] studies escort density operators and generalized Fisher information measures. Also, some mathematical considerations relevant to the present discussion have been given in Ref. [20].

We will analyze here the geometrical approach to statistical mechanics with regard to generalized measures of information, as the one usually denoted as Tsallis' nonextensive $q$-entropy $[15,16]$

$$
S_{q}(\hat{\rho}) \equiv k_{B} \frac{1-\operatorname{Tr} \hat{\rho}^{q}}{q-1} \quad(q \in \mathbb{R})
$$

for which we will show how to derive the metric tensor and other geometric quantities in the space of parameters that define the state of the system, $\hat{\rho}$. Our considerations will refer to a generalized thermostatistical context characterized by a given nonextensive index $q$ and by a given prescription for the expectation values of relevant magnitudes.

The paper is organized as follows: in Section 2 we summarize some basic results for the density operator in a generalized statistical framework. Section 3 is devoted to exhibit some standard results on the geometrical aspects of the space of parameters, while in Section 4 we present an extension of that study to a generalized statistical framework. For an arbitrary value of the nonextensivity index $q$, we obtain the metric tensor of the parameters' space and discuss the meaning of various alternative ways of rewriting the metric tensor. Then, we calculate the scalar curvature in terms of the pseudo-partition function (given by Eq. (4) below), the generalized mean values, and their derivatives with respect to the thermodynamic parameters. As an application we consider the ideal gas case in Section 5. Finally, some conclusions are remarked in Section 6.

\section{The density operator for generalized statistics' OLM-version}

The density operator that maximizes Tsallis nonextensive $q$-entropy (1) for a given positive value of $q$ is written, within the optimized Lagrange multipliers (OLM) formalism [23], as

$$
\hat{\rho}=\frac{1}{\bar{Z}_{q}} e_{q}\left(-\sum_{i=1}^{r} \beta^{i}\left(\hat{F}_{i}-m_{i}\right)\right),
$$

where $m_{1}, \ldots, m_{r}$ are the generalized expectation values of $r$ quantum operators. They are considered to be known as prior information and are given by the following generalized prescription $[21,22]$

$$
m_{i}=\left\langle\hat{F}_{i}\right\rangle_{q} \equiv \frac{\operatorname{Tr} \hat{\rho}^{q} \hat{F}_{i}}{\operatorname{Tr} \hat{\rho}^{q}}, \quad i=1, \ldots, r
$$

(for the sake of notational simplicity, we do not use along this article any label $q$ for $\hat{\rho}$ nor $m_{i}$ despite the fact that they do depend on that index; we keep, however, the subindex in quantities such as $S_{q}$ and $\bar{Z}_{q}$ ). The set of Lagrange multipliers $\left\{\beta^{1}, \ldots, \beta^{r}\right\}$ is such that it fits restrictions (3) in the procedure of constrained extremization of $S_{q}$ when one is working within the OLM formalism [21], i.e., the restrictions are rephrased as centered mean quantities, $\operatorname{Tr}\left[\hat{\rho}^{q}\left(\hat{F}_{i}-m_{i}\right)\right]=0$. This formalism is an alternative to the TMP one of Ref. [22]. It has been established [23,24] that, within OLM method, the $\left\{\beta^{i}\right\}$ correspond to the physical intensive parameters (e.g. the multiplier associated with the Hamiltonian operator, $\hat{F}_{1}=\hat{H}$, is $\beta^{1}=\beta=1 /\left(k_{B} T\right)$ where 
$T$ is the physical temperature of the system). In this framework, the pseudo-partition function

$$
\bar{Z}_{q} \equiv \operatorname{Tr} e_{q}\left(-\sum_{i=1}^{r} \beta^{i}\left(\hat{F}_{i}-m_{i}\right)\right)
$$

is defined in such a form that the density operator is automatically normalized, i.e., $\operatorname{Tr} \hat{\rho}=1$. Notice that no Lagrange multiplier associated with this normalization condition for $\hat{\rho}$ is explicitly mentioned in writing down the equilibrium density matrix; instead, here we choose to introduce the pseudo-partition function in the expression for $\hat{\rho}$. In all these expressions, $e_{q}(x)$ stands for the $q$-exponential function

$$
e_{q}(x) \equiv[1+(1-q) x]_{+}^{1 /(1-q)}
$$

with $[X]_{+} \equiv X \Theta(X)$, being $\Theta$ the Heaviside step function. Finally, the "extensive limit" corresponds to the situation $q \rightarrow 1$, and $q-1$ becomes a measure of the degree of entropic nonextensivity.

\section{Geometrical aspects of parameters' space in standard statistics}

One of the key ideas for achieving our aims is to represent the equilibrium density operator, and related quantities, in an $r$-dimensional space whose coordinate axes correspond to the parameters $\beta^{1}, \ldots, \beta^{r}$ (called the statistical temperatures). These are associated to the operators $\hat{F}_{1}, \ldots, \hat{F}_{r}$, the mean values of which are assumed to constitute the known prior information. One can consider "neighbor" quantum states in the $r$-dimensional space of parameters and, from a notion of distance between states, derive the metric tensor. Janyszek and other authors [2,6-9] have done so for the equilibrium density operator that maximizes von Neumann-Shannon-Gibbs entropy, i.e., $\hat{\rho}=Z^{-1} \exp \left(-\sum \beta^{i} \hat{F}_{i}\right)$ with $Z=\operatorname{Tr} \exp \left(-\sum \beta^{i} \hat{F}_{i}\right)$. For the case of commuting operators $\hat{F}_{i}$, the metric tensor can be defined as

$$
g_{i j}(\beta)=\left\langle\partial_{j} \ln \hat{\rho} \partial_{i} \ln \hat{\rho}\right\rangle, \quad i, j=1, \ldots, r,
$$

where $\langle\ldots\rangle \equiv \operatorname{Tr}(\hat{\rho} \ldots)$ and $\partial_{i} \equiv \partial / \partial \beta^{i}$. For the case of noncommuting operators, this expression should at least be symmetrized. However a different derivation can be adopted, introducing the notion of information distance between two quantum states, say $\hat{\rho}$ and $\hat{\sigma}$, through the relative entropy or information gain (also known as Kullback-Leibler divergence), which is given by

$$
K(\hat{\rho} \| \hat{\sigma})=\operatorname{Tr} \hat{\rho}(\ln \hat{\rho}-\ln \hat{\sigma}) .
$$

The derivation of the metric tensor is as follows (see, for instance, Ref. [6]): first a symmetrical (squared) information distance is constructed, as the sum $D^{2}(\hat{\rho}, \hat{\sigma}) \equiv K(\hat{\rho} \| \hat{\sigma})+K(\hat{\sigma} \| \hat{\rho})$; this quantity is evaluated for a pair of equilibrium density operators, $\hat{\rho}(\{\beta\})$ and $\hat{\rho}(\{\beta+\Delta \beta\})$, which are neighbor states in the parameters' space. The ensuing expression is then expanded as a Taylor series for small displacements $\Delta \beta^{i}$ in all directions, resulting in vanishing zeroth and first order contributions. The nonvanishing second order terms thus allow for the definition of the metric tensor from the local square distance $\mathrm{d} D^{2}=g_{i j} \mathrm{~d} \beta^{i} \mathrm{~d} \beta^{j}$ as

$$
\begin{aligned}
g_{i j}(\beta) & =-\left\langle\partial_{j} \partial_{i} \ln \hat{\rho}\right\rangle \\
& =\partial_{j} \partial_{i} \ln Z
\end{aligned}
$$

or, recalling that $\left\langle\hat{F}_{i}\right\rangle=-\partial_{i} \ln Z$,

$$
g_{i j}(\beta)=-\partial_{j}\left\langle\hat{F}_{i}\right\rangle \text {. }
$$

For classical systems or commuting operators, the definitions for $g_{i j}$ given by Eqs. (6) and (8) do coincide. In these cases it is also seen that the fundamental tensor is related to thermodynamic fluctuations, as the component $i j$ of the tensor corresponds to the covariance or second moment of the pair of operators $\hat{F}_{i}$ and $\hat{F}_{j}$ :

$$
g_{i j}=\left\langle\left(\hat{F}_{i}-\left\langle\hat{F}_{i}\right\rangle\right)\left(\hat{F}_{j}-\left\langle\hat{F}_{j}\right\rangle\right)\right\rangle .
$$

Let us comment that for noncommuting operators an integral expression for the covariances is introduced, based on this connection with the tensor $g_{i j}$ - see Ref. [6] for details. Finally, we notice that the metric tensor, 
being constructed from second derivatives of the Kullback-Leibler divergence, corresponds to the Fisher information matrix.

The metric tensor is one of the key ingredients in the geometric approach to thermostatistics. All the different viewpoints summarized here, Eqs. (8)-(11), have been worked out by many authors in the framework of standard $(q=1)$ statistics, and a number of applications to different physical models have been analyzed in this way. We present in the following section the formalism leading to analogous results for generalized statistics.

\section{Extension of geometrical concepts to nonextensive statistical frameworks}

\subsection{The fundamental tensor}

In the wake of the approach presented in Ref. [6] and working here along similar lines, we consider a generalization of the definition of information gain to nonextensive settings of index $q \neq 1$. The quantum $q$-divergence [25-27] for two normalized operators $\hat{\rho}$ and $\hat{\sigma}$ has been defined as

$$
K_{q}(\hat{\rho} \| \hat{\sigma})=\frac{1}{1-q}\left(\operatorname{Tr} \hat{\rho}-\operatorname{Tr} \hat{\rho}^{q} \hat{\sigma}^{1-q}\right)
$$

generalizing the quantum Kullback-Leibler divergence or relative entropy, Eq. (7). $K_{q}$ can also be written in the following equivalent form:

$$
K_{q}(\hat{\rho} \| \hat{\sigma})=\operatorname{Tr} \hat{\rho}^{q}\left(\ln _{q} \hat{\rho}-\ln _{q} \hat{\sigma}\right),
$$

where the so-called $q$-logarithm $\ln _{q} x \equiv\left(x^{1-q}-1\right) /(1-q)$ for $x \geqslant 0$ (excluding $x=0$ if $q>1$ ), represents the inverse function of $e_{q}(x)$. It is straightforward to show that $e_{q}(x)$ and $\ln _{q}(x)$ tend to the exponential and logarithm functions, respectively, for $q$ sufficiently near 1 (standard limit).

The next step in our derivation is to extend the concept of (squared) information distance, $D^{2}(\hat{\rho}, \hat{\sigma})$. One can see that $K_{q}(\hat{\rho} \| \hat{\sigma}) \neq K_{q}(\hat{\sigma} \| \hat{\rho})$, except for the particular cases: (i) $q=\frac{1}{2}$ (Hellinger distance, with the conditions $\operatorname{Tr} \hat{\rho}=\operatorname{Tr} \hat{\sigma}=1$ ), and (ii) $q=0$ (since $K_{0}=0$ for any pair of normalized density operators). In general, then, for our purposes we make use of the symmetrized sum

$$
K_{q}(\hat{\rho} \| \hat{\sigma})+K_{q}(\hat{\sigma} \| \hat{\rho})
$$

as information measure. From a Taylor expansion of this quantity around a certain set of values for the parameters $\left\{\beta^{i}\right\}$ (i.e., a given point in the $r$-dimensional $\beta$-space), that characterize the state of the system, we determine the form of the fundamental tensor in a generalized context (in fact, we determine a family of metrics which are characterized by the single index $q$, with $q \in \mathbb{R}^{+}$). Let us then construct the first few terms of the associated Taylor series, taking $\hat{\rho}=\hat{\rho}(\beta)$ and $\hat{\sigma}=\hat{\rho}(\beta+\Delta \beta)$ :

- The zeroth-order term is identically zero since $K_{q}(\hat{\rho} \| \hat{\sigma})=0$ if and only if $\hat{\rho}=\hat{\sigma}$. This was also the case for the standard $(q=1)$ context.

- From Eqs. (12) and (13), the first-order term vanishes identically as in the standard case. This is a consequence of the shape adopted for the symmetrized information gain. In other words, the explicit form of the density operator does not play any role at this level.

- From the second-order terms in our Taylor expansion of the symmetric information measure (13), we obtain the following result, which we interpret as the generalized fundamental tensor within the nonextensive picture:

$$
\begin{aligned}
g_{i j}^{(q)} & =q \operatorname{Tr}\left(\hat{\rho}^{-1} \frac{\partial \hat{\rho}}{\partial \beta^{j}} \frac{\partial \hat{\rho}}{\partial \beta^{i}}\right) \\
& =-q \operatorname{Tr}\left(\hat{\rho} \frac{\partial^{2} \ln \hat{\rho}}{\partial \beta^{j} \partial \beta^{i}}\right),
\end{aligned}
$$

where $\hat{\rho}(\beta)$ is the OLM density matrix (2). We notice that this result can also be regarded as the $i j$ element of the generalized Fisher information (GFI) matrix $G^{(q)}=\left(g_{i j}^{(q)}\right)$ (see, for instance, Ref. [28] and references 
therein). Interestingly enough, from Eqs. (6), (8), (14) and (15) we gather that this quantity-when expressed as a trace - exhibits shape invariance, irrespective of the value of $q$. Indeed, the form of the GFI matrix is seen to be the same for any value of the nonextensivity index $q$, and it also coincides with that of the standard case. Differences arise, of course, when one introduces explicitly the actual density operator, which may correspond either to the extensive or to the nonextensive scenario.

Summing up, we have found the fundamental tensor of the space of parameters within a nonextensive statistical framework, in terms of first logarithmic derivatives of the generalized density operator, $g_{i j}^{(q)}=q \operatorname{Tr}\left(\hat{\rho} \partial_{j} \ln \hat{\rho} \partial_{i} \ln \hat{\rho}\right)$, as well as in terms of second derivatives of $\ln \hat{\rho}$, Eq. (15), with $\hat{\rho}$ given as in Eq. (2). Notice that, in both expressions, the standard logarithm function is involved.

It is instructive to look at alternative formulations of the results already obtained. After a little algebra we can rewrite each of the expressions mentioned previously, in terms of variations of the logarithm of the pseudo-partition function:

$$
g_{i j}^{(q)}=q\left\{-\partial_{j} m_{i}-(q-1) \bar{Z}_{q}^{2(q-1)} \operatorname{Tr}\left[\hat{\rho}^{2 q-1}\left(\delta_{q} \hat{F}_{j}-\partial_{j} \ln \bar{Z}_{q}\right)\left(\delta_{q} \hat{F}_{i}-\partial_{i} \ln \bar{Z}_{q}\right)\right]\right\}
$$

and

$$
g_{i j}^{(q)}=q\left\{-\partial_{j} \ln \bar{Z}_{q} \partial_{i} \ln \bar{Z}_{q}+\bar{Z}_{q}^{2(q-1)} \operatorname{Tr}\left[\hat{\rho}^{2 q-1}\left(\delta_{q} \hat{F}_{j}-\partial_{j} \ln \bar{Z}_{q}\right)\left(\delta_{q} \hat{F}_{i}-\partial_{i} \ln \bar{Z}_{q}\right)\right]\right\},
$$

respectively, where the generalized deviation operators are defined as $\delta_{q} \hat{F}_{i} \equiv \hat{F}_{i}-m_{i}$ with $m_{i}$ given by Eq. (3). Comparing these expressions we immediately obtain a useful (symmetric) relation for the first derivative of a given mean value with respect to any of the Lagrange parameters that writes

$$
\partial_{j} m_{i}=\partial_{i} m_{j}=\partial_{j} \ln \bar{Z}_{q} \partial_{i} \ln \bar{Z}_{q}-q \bar{Z}_{q}^{2(q-1)} \operatorname{Tr}\left[\hat{\rho}^{2 q-1}\left(\delta_{q} \hat{F}_{j}-\partial_{j} \ln \bar{Z}_{q}\right)\left(\delta_{q} \hat{F}_{i}-\partial_{i} \ln \bar{Z}_{q}\right)\right]
$$

from which the components of the metric tensor can finally be cast in compact form as

$$
g_{i j}^{(q)}=-\partial_{j} m_{i}-(q-1) \partial_{j} \ln \bar{Z}_{q} \partial_{i} \ln \bar{Z}_{q} .
$$

This covariant tensor of rank 2 is seen to be symmetric (by use of Eq. (18)). Its conjugate or reciprocal tensor, $g^{(q)} i j$, is a symmetric tensor of contravariant valence two.

Yet another form of representing the $q$-metric tensor involves the use of fluctuations, as in Eq. (11). This point has been discussed with some detail in Ref. [20], where generalized expressions for operator variance and covariance have also been considered.

We see from (19) that, in order to evaluate the generalized metric tensor for a given system, we need the logarithmic derivatives of the pseudo-partition function with respect to the Lagrange parameters, as well as the behavior of the generalized expectation values of the relevant operators in $\beta$-space. We end up by remarking that the standard limit for the fundamental tensor is correctly obtained in all the equivalent forms developed in this section.

\subsection{The scalar curvature}

Let us now address the computation of the curvature of the Riemannian space of parameters. We remind that in the extensive $q=1$ framework [6] the metric tensor $G=\left(g_{i j}\right)$ corresponds to the matrix of second partial derivatives of the function $f(\{\beta\})=\ln Z$ with respect to every pair of parameters, i.e., the Hessian matrix of $\ln Z, G=H(\ln Z)$ (see Eq. (9)). As a consequence, the curvature tensor and the scalar curvature of the Riemann space, being constructed from derivatives of the elements of $G$, adopt a relatively simple form. Using Eq. (9), the results are the following [6]:

$$
R_{i j k l}=\frac{1}{4} g^{m n}\left(f_{, m i l} f_{, n j k}-f_{, m i k} f_{, n j l}\right), \quad f \equiv \ln Z
$$

for the curvature tensor, where $f_{, i} \equiv \partial_{i} f=\partial \ln Z / \partial \beta^{i}$ and so on, and

$$
R=\frac{2}{g} R_{1212}, \quad g=|G|
$$


for the scalar curvature, with $g=|H(f)|$. Eq. (21) is valid in the particular case of considering only $r=2$ parameters.

In a nonextensive setting characterized by a given fixed value of $q$, we arrive at more involved expressions that, however, reduce to the corresponding $q \rightarrow 1$ standard limit. For the sake of brevity, we define here

$$
\bar{f} \equiv \ln \bar{Z}_{q}
$$

(employing the standard logarithm) so that the Riemann-Christoffel tensor reads [10,11]

$$
\begin{aligned}
R_{i j k l}^{(q)}= & -(q-1)\left(\bar{f}_{, i k} \bar{f}_{, j l}-\bar{f}_{, i l} \bar{f}_{, j k}\right)-g^{(q) s t}\left\{\frac{1}{4}\left(m_{s, j l} m_{t, i k}-m_{s, j k} m_{t, i l}\right)\right. \\
& \left.+\frac{1}{2}(q-1)\left[\bar{f}_{, s}\left(m_{t, i k} \bar{f}_{, j l}-m_{t, i l} \bar{f}_{, j k}\right)+\left(\bar{f}_{, i k} m_{s, j l}-\bar{f}_{, i l} m_{s, j k}\right) \bar{f}_{, t}\right]+(q-1)^{2}\left(\bar{f}_{, i k} \bar{f}_{, j l}-\bar{f}_{, i l} \bar{f}_{, j k}\right) \bar{f}_{, s} \bar{f}_{, t}\right\}
\end{aligned}
$$

(with the notation $m_{s, i j} \equiv \partial_{j} \partial_{i} m_{s}$ ). This is one of our main results in the present effort. The $m_{i}$ correspond to generalized expectation values, and $\bar{f}$ stands for the logarithm of the pseudo-partition function. Apart from those $q$-dependent quantities, we appreciate in Eq. (22) the fact that: (i) some new terms, of order $(q-1)$ and $(q-1)^{2}$, emerge in the nonextensive framework with respect to the standard result (20), and (ii) the metric tensor itself contains an explicit contribution with $(q-1)$, arising from Eq. (19). It can be checked that the only terms that survive on letting $q \rightarrow 1$, exactly correspond to Eq. (20).

We can now compute, by appropriate contractions, first the Ricci tensor $R_{j k}^{(q)}=g^{(q) i l} R_{i j k l}^{(q)}$ and then the scalar curvature $R^{(q)}=g^{(q) j k} R_{j k}^{(q)}$. A combinatorial analysis shows that, for an $r$-dimensional Riemann space, the number of independent components of the $(4,0)$-type Riemann-Christoffel curvature tensor is $r^{2}\left(r^{2}-1\right) / 12$, due to symmetry reasons [11]. At this point, in order to fix ideas and also for application purposes, we address the particular case of $r=2$ Lagrange parameters characterizing the system. In this case it suffices merely to obtain an expression for $R_{1212}^{(q)}$ and, then, the desired scalar curvature $R^{(q)}$ can be computed making use of a relation of the form given in Eq. (21) (notice that this equation is valid for any two-dimensional metric). On the one hand, we calculate the $2 \times 2$-determinant $g^{(q)} \equiv\left|G^{(q)}\right|$ :

$$
\begin{aligned}
g^{(q)} & =\left|\left(\begin{array}{ll}
m_{1,1} & m_{1,2} \\
m_{2,1} & m_{2,2}
\end{array}\right)+(q-1)\left(\begin{array}{cc}
\bar{f}_{, 1}^{2} & \bar{f}_{, 1} \bar{f}_{, 2} \\
\bar{f}_{, 2} \bar{f}_{, 1} & \bar{f}_{, 2}^{2}
\end{array}\right)\right| \\
& =\left|\begin{array}{ll}
m_{1,1} & m_{1,2} \\
m_{2,1} & m_{2,2}
\end{array}\right|+(q-1)\left\{\bar{f}_{, 1}\left|\begin{array}{ll}
\bar{f}_{, 1} & \bar{f}_{, 2} \\
m_{2,1} & m_{2,2}
\end{array}\right|-\bar{f}_{, 2}\left|\begin{array}{ll}
\bar{f}_{, 1} & \bar{f}_{, 2} \\
m_{1,1} & m_{1,2}
\end{array}\right|\right\}
\end{aligned}
$$

and, on the other hand, we compute separately each term appearing in Eq. (22), for $(i j k l)=(1212)$ :

(1) the term $-(q-1)\left(\bar{f}_{, 11} \bar{f}_{, 22}-\bar{f}_{, 12} \bar{f}_{, 21}\right)$ equals

$$
-(q-1)\left|\begin{array}{ll}
\bar{f}_{, 11} & \bar{f}_{, 12} \\
\bar{f}_{, 21} & \bar{f}_{, 22}
\end{array}\right|,
$$

which is proportional to $|H(\bar{f})|$;

(2) the term $-\frac{1}{4} g^{(q) s t}\left(m_{s, 22} m_{t, 11}-m_{s, 21} m_{t, 12}\right)$ equals $1 /\left(4 g^{(q)}\right)$ times

$$
\left|\begin{array}{lll}
m_{1,1} & m_{1,2} & m_{2,2} \\
m_{1,11} & m_{1,12} & m_{1,22} \\
m_{1,12} & m_{1,22} & m_{2,22}
\end{array}\right|+(q-1)\left|\begin{array}{lll}
\bar{f}_{, 1}^{2} & \bar{f}_{, 1} \bar{f}_{, 2} & \bar{f}_{, 2}^{2} \\
m_{1,11} & m_{1,12} & m_{1,22} \\
m_{1,12} & m_{1,22} & m_{2,22}
\end{array}\right| .
$$


(3) The term $-(q-1) \frac{1}{2} g^{(q) s t}\left[\bar{f}_{, s}\left(m_{t, 11} \bar{f}_{, 22}-m_{t, 12} \bar{f}_{, 21}\right)+\bar{f}_{, t}\left(m_{s, 22} \bar{f}_{, 11}-m_{s, 21} \bar{f}_{, 12}\right)\right]$ equals $(q-1) /\left(2 g^{(q)}\right)$ times

$$
\begin{aligned}
& \left|\begin{array}{ll}
\bar{f}_{, 1} & \bar{f}_{, 2} \\
m_{2,1} & m_{2,2}
\end{array}\right|\left(\left|\begin{array}{ll}
\bar{f}_{, 11} & \bar{f}_{, 12} \\
m_{1,21} & m_{1,22}
\end{array}\right|-\left|\begin{array}{ll}
\bar{f}_{, 21} & \bar{f}_{, 22} \\
m_{1,11} & m_{1,12}
\end{array}\right|\right) \\
& \quad-\left|\begin{array}{cc}
\bar{f}_{, 1} & \bar{f}_{, 2} \\
m_{1,1} & m_{1,2}
\end{array}\right|\left(\left|\begin{array}{ll}
\bar{f}_{, 11} & \bar{f}_{, 12} \\
m_{2,21} & m_{2,22}
\end{array}\right|-\left|\begin{array}{cc}
\bar{f}_{, 21} & \bar{f}_{, 22} \\
m_{2,11} & m_{2,12}
\end{array}\right|\right) .
\end{aligned}
$$

(4) The term $-(q-1)^{2}\left(\bar{f}_{, 11} \bar{f}_{, 22}-\bar{f}_{, 12} \bar{f}_{, 21}\right) g^{(q) s t} \bar{f}_{, s} \bar{f}_{, t}$ equals $(q-1)^{2} / g^{(q)}$ times

$$
\left|\begin{array}{ll}
\bar{f}_{, 11} & \bar{f}_{, 12} \\
\bar{f}_{, 21} & \bar{f}_{, 22}
\end{array}\right|\left\{\bar{f}_{, 1}\left|\begin{array}{ll}
\bar{f}_{, 1} & \bar{f}_{, 2} \\
m_{2,1} & m_{2,2}
\end{array}\right|-\bar{f}_{, 2}\left|\begin{array}{ll}
\bar{f}_{, 1} & \bar{f}_{, 2} \\
m_{1,1} & m_{1,2}
\end{array}\right|\right\} \text {. }
$$

Thus $R_{1212}^{(q)}$ is obtained as the sum of the contributions in expressions (24)-(27), with their corresponding prefactors, and with $g^{(q)}$ given by Eq. (23). Notice that we have arranged all the second order determinants as Jacobian ones, involving the two-parameters functions $m_{1}, m_{2}$, and $\bar{f}$ as well as their first derivatives with respect to both parameters. For brevity, we will denote the Jacobian determinant $\partial(\phi, \psi) / \partial\left(\beta^{1}, \beta^{2}\right)$ simply as $J(\phi, \psi)$. Also, the Hessian determinant of $\bar{f}$ is to be denoted as $H(\bar{f})$ wherever it appears (see Eq. (24)). Summing up, the generalized scalar curvature of 2D-space becomes

$$
\begin{aligned}
& R^{(q)}=\frac{2}{\left(g^{(q)}\right)^{2}}\left\{\frac{1}{4}\left|\begin{array}{lll}
m_{1,1} & m_{1,2} & m_{2,2} \\
m_{1,11} & m_{1,12} & m_{1,22} \\
m_{1,12} & m_{1,22} & m_{2,22}
\end{array}\right|+(q-1)\left[\frac{1}{4}\left|\begin{array}{lll}
\bar{f}_{, 1}^{2} & \bar{f}_{, 1} \bar{f}_{, 2} & \bar{f}_{, 2}^{2} \\
m_{1,11} & m_{1,12} & m_{1,22} \\
m_{1,12} & m_{1,22} & m_{2,22}
\end{array}\right|\right.\right. \\
& \left.\left.-J\left(m_{1}, m_{2}\right)|H(\bar{f})|+\frac{1}{2}\left[J\left(\bar{f}, m_{2}\right)\left(J\left(\bar{f}_{, 1}, m_{1,2}\right)-J\left(\bar{f}_{, 2}, m_{1,1}\right)\right)-J\left(\bar{f}, m_{1}\right)\left(J\left(\bar{f}_{, 1}, m_{2,2}\right)-J\left(\bar{f}_{, 2}, m_{2,1}\right)\right)\right]\right]\right\}
\end{aligned}
$$

with

$$
g^{(q)}=J\left(m_{1}, m_{2}\right)+(q-1)\left[\bar{f}_{, 1} J\left(\bar{f}, m_{2}\right)-\bar{f}_{, 2} J\left(\bar{f}, m_{1}\right)\right] .
$$

In these expressions it is not difficult to recognize the extensive limit, that coincides with the result given, for instance, in Ref. [6]. Finally, one could regroup all the contributions to $R^{(q)}$ and analyze the ensuing departure from the extensive situation, which may eventually shed some light on the behavior of the system under study. Those terms that do not contain any explicit factor $(q-1)^{n}$, or "zeroth-order terms", are clearly the ones that survive in the limit $q \rightarrow 1$, while higher order terms in $(q-1)$ represent the successive corrections to the extensive case, when $q$ is close to one.

\section{The ideal gas case}

We address here a classical ideal system consisting of $N$ free particles in contact with a heat bath at temperature $T$ and pressure $P$ through a movable wall (the volume is variable). Considering the $P-T$, or Boguslavski, distribution, the standard partition function (usually denoted as $Y_{N}(P, T)$ in textbooks) is given by

$$
Z_{1}(\alpha, \beta)=\frac{1}{N ! h^{3 N}} \int_{0}^{\infty} \mathrm{d} V \int \mathrm{d}^{3 N} q \mathrm{~d}^{3 N} p \mathrm{e}^{-\alpha V-\beta H}=\alpha^{-(N+1)}\left(\frac{2 \pi m}{h^{2} \beta}\right)^{3 N / 2},
$$


where $H$ denotes the free particle Hamiltonian for $N$ particles of mass $m$, and the intensive parameters are taken to be $\alpha=P / k T$ and $\beta=1 / k T$.

One can employ Eq. (30) as a starting point to calculate the nonextensive thermodynamical magnitudes of the system, by recourse to integral representations based on the definition of the Euler gamma function (see, for instance, the appendix in Ref. [29] for details and a discussion regarding its validity). Performing the socalled Hilhorst transform, which is applicable if the nonextensivity index $q$ is greater than 1, the generalized pseudo-partition function can be written in the form

$$
\bar{Z}_{q}(\alpha, \beta)=Z_{1}(\alpha, \beta) \frac{\Gamma\left(1 /(q-1)-\frac{5}{2} N-1\right)(1 /(q-1))^{(5 / 2) N+1}}{\Gamma(1 /(q-1))\left[1-(q-1) I_{q}\right]^{1 /(q-1)-(5 / 2) N-1}},
$$

where for brevity we have defined the quantity $I_{q}=\alpha\langle V\rangle_{q}+\beta\langle H\rangle_{q} \equiv \alpha V_{q}+\beta U_{q}$, which is a convenient combination of the intensive parameters and corresponding generalized mean values for volume and energy, respectively. The range of $q$ for which Eq. (31) is valid is given by $0<q-1<\left(\frac{5}{2} N+1\right)^{-1}$. Performing the same sort of real integral representation as before, one can compute the $q$-expectation values of the relevant operators. The following interesting result ensues:

$$
V_{q}=\frac{N+1}{\alpha} \text { and } U_{q}=\frac{3 N}{2 \beta},
$$

while $I_{q}=\frac{5}{2} N+1$. It is remarkable that these results are independent of the value of the index $q$ (as long as it belongs to the range of validity of the integral representation employed). Also, for later purposes, we notice that $U_{q}$ does not depend on the pressure, while $V_{q}$ is a function of $\alpha$ only. In both expressions, $N$ is the (fixed) number of particles that constitute the system under study; note that in the "equation of state" there is a factor $N+1$ instead of $N$ as we have not taken the thermodynamic limit which allows for that replacement.

With these ingredients we can compute the generalized metric tensor and other geometrical quantities of interest defined in Section 4. From Eqs. (31) and (32), for the $q$-metric tensor (19) we obtain the following $2 \times 2$ symmetric matrix

$$
\left(g_{i j}^{(q)}\right)=\left(\begin{array}{ll}
\frac{N+1}{\alpha^{2}} & 0 \\
0 & \frac{3 N}{2 \beta^{2}}
\end{array}\right)-(q-1)\left(\begin{array}{ll}
\left(\frac{N+1}{\alpha}\right)^{2} & \frac{3 N(N+1)}{2 \alpha \beta} \\
\frac{3 N(N+1)}{2 \alpha \beta} & \left(\frac{3 N}{2 \beta}\right)^{2}
\end{array}\right)
$$

for $1<q<1+\left(\frac{5}{2} N+1\right)^{-1}$. The limit $q \rightarrow 1^{+}$gives the correct diagonal result already obtained by other authors (Refs. [7,8], for instance). Here, the changes introduced by the nonextensive treatment appear as contributions of order $(q-1)$ : a dependence with $N^{2}$ appears since we have not considered thermodynamic magnitudes per particle (as Brody and Rivier, for instance, do). The $2 \times 2$-determinant of matrix (33) acquires a simple form in terms of the intensive parameters $\alpha$ and $\beta$, and the number of particles $N$ :

$$
g^{(q)}=\frac{3 N(N+1)}{2 \alpha^{2} \beta^{2}}\left[1-(q-1)\left(\frac{5}{2} N+1\right)\right] .
$$

We compute explicitly the expressions in (24)-(27) (with their corresponding prefactors) and arrive, respectively, at

$$
\begin{aligned}
& \frac{(q-1)}{g^{(q)}}\left(\frac{3 N(N+1)}{2 \alpha^{2} \beta^{2}}\right)^{2} \times\left\{-1+(q-1)\left(\frac{5}{2} N+1\right)\right\}, \\
& \frac{(q-1)}{g^{(q)}}\left(\frac{3 N(N+1)}{2 \alpha^{2} \beta^{2}}\right)^{2} \times\{-1\},
\end{aligned}
$$




$$
\begin{aligned}
& \frac{(q-1)}{g^{(q)}}\left(\frac{3 N(N+1)}{2 \alpha^{2} \beta^{2}}\right)^{2} \times\{2\}, \\
& \frac{(q-1)}{g^{(q)}}\left(\frac{3 N(N+1)}{2 \alpha^{2} \beta^{2}}\right)^{2} \times\left\{-(q-1)\left(\frac{5}{2} N+1\right)\right\} .
\end{aligned}
$$

Adding these four terms and multiplying by the factor $2 / g^{(q)}$ we are led to $R^{(q)}$. The computation of the generalized scalar curvature in this example yields the outcome: $R^{(q)}=0$. It is noticeable that this result: (i) is independent of the intensive parameters $\alpha$ and $\beta$, and (ii) is also independent of the nonextensivity index $q$. We can then assert that in this case the outcome for the curvature in our geometrical approach, performed within the framework of a nonextensive statistical setting, agrees with the developments obtained by different authors within the structures of the extensive formalism - see, for instance, Refs. [7,8].

\section{Concluding remarks}

We have discussed geometrical aspects of the space of parameters for a physical system, within the framework of a nonextensive statistical setting of fixed index $q$. For that purpose, we have employed a generalization of the quantum Kullback-Leibler divergence as information gain. In particular, we have derived the pertinent generalized metric tensor which is connected with Fisher's information matrix. Our main findings in the present effort are represented by Eqs. (19) and (28), which pave the way for deriving the metric and curvature tensors from a generalized partition function for a fixed value of the nonextensivity index.

The studies addressed here are relevant in the investigation of critical behavior and phase transitions in thermodynamic systems. Work on such sort of applications for interacting systems, using generalized information measures, is currently in progress.

Finally, we remark that the use of alternative measures of distance between pairs of density operators in the space of thermodynamic parameters could yield an alternative method-perhaps a more sensible one- to detect thermodynamic changes in certain physical systems applying the geometric machinery discussed in the present effort. To mention just one such possibility, the Jensen-Shannon (JS) divergence can be used to give a measure of distance between two probability distributions. A generalization of the JS divergence in the framework of Tsallis statistical mechanics has been investigated by Lamberti and co-workers [30,31], who obtained a monoparametric family of metrics. This and other alternatives will be the subject of future work.

\section{Acknowledgments}

The authors acknowledge financial support from CONICET and UNLP, Argentina. MP also acknowledges ANPCyT (PICT No. 03-11903/2002), Argentina.

\section{References}

[1] F. Weinhold, J. Chem. Phys. 63 (1975) 2479 2484,2488; F. Weinhold, J. Chem. Phys. 65 (1976) 559.

[2] G. Ruppeiner, Phys. Rev. A 20 (1979) 1608; G. Ruppeiner, Rev. Mod. Phys. 67 (1995) 605 Erratum 68 (1996) 313.

[3] R. Mrugała, Physica 125A (1984) 631.

[4] R.S. Ingarden, Tensor NS 30 (1976) 201.

[5] H. Janyszek, Rep. Math. Phys. 24 (1986) 1,11.

[6] H. Janyszek, R. Mrugała, Phys. Rev. A 39 (1989) 6515.

[7] H. Janyzsek, J. Phys. A 23 (1990) 477.

[8] D. Brody, N. Rivier, Phys. Rev. E 51 (1995) 1006.

[9] W. Janke, D.A. Johnston, R. Kenna, Phys. Rev. E 67 (2003) 046106; W. Janke, D.A. Johnston, R. Kenna, Physica A 336 (2004) 181.

[10] P. Le Corbeiller, Sci. Am. 191 (5) (1954) 80.

[11] L.A. Santaló, Vectores y Tensores con sus Aplicaciones, EUDEBA, Buenos Aires, 1981, 12a. ed. 
[12] D. Poole, Algebra Lineal: Una Introducción Moderna (Linear Algebra: A Modern Introduction), Thomson, Mexico, 2004.

[13] C.R. Rao, Bull. Calcutta Math. Soc. 37 (1945) 81.

[14] S. Amari, Differential-Geometrical Methods in Statistics, Lecture Notes in Statistics, vol. 28, Springer, New York, 1985;

S. Amari, H. Nagaoka, Methods of Information Geometry, AMS and Oxford University Press, London, 2000.

[15] C. Tsallis, J. Stat. Phys. 52 (1988) 479.

[16] A periodically updated bibliography on nonextensive thermostatistics can be found in the URL /http://tsallis.cat.cbpf.br/biblio.htm〉.

[17] S. Abe, Phys. Rev. E 68 (2003) 031101.

[18] R. Trasarti-Battistoni, cond-mat/0203536.

[19] J. Naudts, Open Sys. Inf. Dyn. 12 (2005) 13.

[20] M. Portesi, A. Plastino, F. Pennini, Physica A 365 (2006) 173.

[21] S. Martínez, F. Nicolás, F. Pennini, A. Plastino, Physica A 286 (2000) 489.

[22] C. Tsallis, R.S. Mendes, A.R. Plastino, Physica A 261 (1998) 534.

[23] S. Abe, S. Martínez, F. Pennini, A. Plastino, Phys. Lett. A 281 (2001) 126.

[24] S. Abe, Physica A 300 (2001) 417.

[25] P.W. Lamberti, M.T. Martín, A. Plastino, O.A. Rosso, Physica A 334 (2004) 119.

[26] S. Abe, Phys. Rev. A 68 (2003) 032302.

[27] S. Abe, Physica A 344 (2004) 359.

[28] M. Tegmark, A. Taylor, A. Heavens, Astrophys. J. 480 (1997) 22.

[29] S. Martínez, F. Pennini, A. Plastino, M. Portesi, Physica A 332 (2004) 230.

[30] P.W. Lamberti, A.P. Majtey, Physica A 329 (2003) 81.

[31] A.P. Majtey, P.W. Lamberti, A. Plastino, Physica A 344 (2004) 547. 